\begin{tabular}{|c|c|c|c|}
\hline \multirow{3}{*}{$\begin{array}{r}\text { Case Reports in } \\
\text { Gastroenterology }\end{array}$} & Case Rep Gastroenterol 2019; & & \multirow[b]{2}{*}{$\begin{array}{l}\text { Karger } \\
\text { Opengaccess }\end{array}$} \\
\hline & $\begin{array}{l}\text { DOI: 10.1159/000503897 } \\
\text { Published online: November 1, } 2019\end{array}$ & $\begin{array}{l}\text { (c) } 2019 \text { The Author(s) } \\
\text { Published by S. Karger AG, Basel } \\
\text { www.karger.com/crg }\end{array}$ & \\
\hline & $\begin{array}{l}\text { This article is licensed under the } \\
\text { International License (CC BY-NC) (h } \\
\text { Usage and distribution for commercial }\end{array}$ & $\begin{array}{l}\text { hons Attribution-NonCommercial } 4.0 \\
\text { ger.com/Services/OpenAccessLicense). } \\
\text { uires written permission. }\end{array}$ & \\
\hline
\end{tabular}

\title{
Primary Gastrointestinal Amyloidosis: An Unusual Cause of Acute Intestinal Pseudo-Obstruction
}

\author{
Panu Wetwittayakhlang Pimsiri Sripongpun Sawangpong Jandee \\ Gastroenterology and Hepatology Unit, Division of Internal Medicine, Faculty of Medicine, \\ Prince of Songkla University, Hat Yai, Songkla, Thailand
}

\section{Keywords}

Gastrointestinal amyloidosis · Primary intestinal amyloidosis - Acute intestinal obstruction . Intestinal pseudo-obstruction

\begin{abstract}
Amyloidosis of the gastrointestinal tract is an uncommon disorder characterized by the extracellular deposition of an abnormal fibrillar protein. It is rarely proven by biopsy. Amyloid deposition interferes with organ structure and its function. We report a case of a 64-year-old male who presented with severe colicky pain, unable to pass feces, and progressive abdominal distension for 2 days. Physical examination revealed marked abdominal distension, visible peristalsis, high-pitched hyperactive bowel sounds, and generalized tenderness. Plain abdominal radiograph showed markedly diffuse disproportional dilatation of the small bowel with different heights of air-fluid levels in the same loop. Abdominal computed tomography showed an evidence of small bowel obstruction, which revealed no gross mass or cause of obstruction, but long segment narrowing of the terminal ileum was seen. Ileocolonoscopy showed diffuse edematous mucosa of the ileum without mechanical obstruction but loss of normal bowel peristalsis. A random biopsy of the ileum was performed for pathological diagnosis, which reported extensive deposits of amorphous material within the muscle layers and in the submucosal vessels that stained strongly with Congo red and displayed the typical apple-green birefringence of amyloid protein when viewed under plane polarized light. Serum electrophoretic tests disclosed a monoclonal band of IgG-kappa monoclonal protein. His clinical symptoms improved after receiving chemotherapy with melphalan and prednisolone. Our case illustrated the rare cause of acute intestinal obstruction which mimicked a surgical condition. Primary intestinal amyloidosis should be in a differential diagnosis in patients without a demonstrated cause of obstruction.




\section{Case Reports in Gastroenterology}

Case Rep Gastroenterol 2019;13:462-467 DOI: $10.1159 / 000503897$

www.karger.com/crg

Wetwittayakhlang et al.: Primary Gastrointestinal Amyloidosis: An Unusual Cause of Acute Intestinal Pseudo-Obstruction

\section{Introduction}

Amyloidosis is a rare disorder characterized by the extracellular deposition of an abnormal fibrillar protein which disrupts tissue structure and function. Amyloidosis can be acquired or hereditary and systemic or localized to a single organ, such as the gastrointestinal (GI) tract [1]. There are six types: primary, secondary, hemodialysis-related, hereditary, senile, and localized amyloidosis. Primary amyloidosis (monoclonal immunoglobulin light chains [AL]) is the most common form of amyloidosis. Fifteen percent of patients have multiple myeloma. Secondary (AA) amyloidosis is associated with chronic inflammatory, infectious, and neoplastic disorders. AA amyloidosis affects the GI tract in $60 \%$ of patients, while $1-8 \%$ of patients with AL amyloidosis have GI involvement [2]. Amyloidosis confined only to the GI tract is rare. The deposition of amyloid proteins interferes with organ structure and its function. The clinical manifestations of intestinal amyloidosis may vary from asymptomatic to fetal forms. We report a rare case of biopsy-proven primary light-chain amyloidosis of the small intestine of a patient who initially presented with symptoms of acute small intestinal pseudoobstruction.

\section{Case Presentation}

A 64-year-old male with a medical history of well-controlled type 2 diabetes mellitus presented at the Emergency Department with severe colicky pain, unable to pass feces and flatus, and progressive abdominal distension for 2 days. The patient reported he had previously suffered from diarrhea 3-4 times per day and intermittent mid-abdominal pain for 2 weeks.

On physical examination, blood pressure was $130 / 75 \mathrm{~mm} \mathrm{Hg}$, pulse rate was $90 / \mathrm{min}$, and temperature was $37.2^{\circ} \mathrm{C}$. Abdominal examination revealed markedly abdominal distension, visible peristalsis, high-pitched hyperactive bowel sounds, and generalized tenderness. No hepatosplenomegaly, abdominal mass, or shifting dullness was demonstrated. The remainder of the physical examination was unremarkable.

Initial laboratory investigations showed a leukocyte count of $14.6 \times 10^{3} / \mu \mathrm{L}$, hemoglobin $11.5 \mathrm{~g} / \mathrm{dL}$, platelet count $366 \times 10^{3} / \mu \mathrm{L}$, blood urea nitrogen $34 \mathrm{mg} \%$, serum creatinine 1.0 $\mathrm{mg} \%$, total bilirubin $1.2 \mathrm{mg} / \mathrm{dL}$, alanine transaminase $25 \mathrm{U} / \mathrm{L}$, aspartate transaminase $18 \mathrm{U} / \mathrm{L}$, alkaline phosphatase $90 \mathrm{U} / \mathrm{L}$, and albumin $3.0 \mathrm{~g} \%$, and urinalysis showed absence of proteinuria. Chest radiograph and electrocardiogram showed no cardiomegaly.

Plain abdominal radiograph disclosed markedly diffuse disproportional dilatation of the small bowel with different heights of air-fluid levels in the same loop (Fig. 1). The patient was admitted to the surgical ward for presumed acute small intestinal obstruction. Subsequent abdominal computed tomography showed an evidence of small bowel obstruction, which revealed no gross mass or cause of obstruction but long segment narrowing of the terminal ileum (Fig. 2).

He subsequently underwent limited low-air insufflation ileocolonoscopy, which showed diffuse edematous mucosa of the ileum without intraluminal obstruction, but loss of normal bowel peristalsis was observed during the procedure. A random biopsy of the ileum was performed for pathological diagnosis, which reported extensive deposits of pink amorphous material in all muscle layers and in the submucosal vessels that stained strongly with Congo red and displayed the typical apple-green birefringence of amyloid deposits when viewed under plane polarized light (Fig. 3). The tissue biopsies from the colon were unremarkable.

Serum electrophoretic tests disclosed a monoclonal band of immunoglobulin G kappa, and a quantitative assessment of immunoglobulins demonstrated the following results: IgG 2,250 (normal 700-1,530) mg/dL, IgA 140 (normal 60-420) mg/dL, IgM 85 (normal 60-310) 


\section{Case Reports in Gastroenterology}

Case Rep Gastroenterol 2019;13:462-467 DOI: $10.1159 / 000503897$

(c) 2019 The Author(s). Published by S. Karger AG, Basel www.karger.com/crg

Wetwittayakhlang et al.: Primary Gastrointestinal Amyloidosis: An Unusual Cause of Acute Intestinal Pseudo-Obstruction

$\mathrm{mg} / \mathrm{dL}$, kappa-free light chains 645 (normal 4.0-25.0) mg/L, and lambda-free light chains 28.5 (normal 6.5-38.0) $\mathrm{mg} / \mathrm{L}$. Bone marrow biopsy was performed to exclude multiple myeloma and the results showed that the proportion of plasma cells was $5 \%$. Thus, primary light-chain amyloidosis of the small intestine was the diagnosis.

The patient received conservative treatments by decompression and nasogastric suction followed by melphalan and dexamethasone. His clinical symptoms improved after the second cycle of chemotherapy. However, he refused to receive autologous stem cell transplantation due to his old age and potential high-risk for transplant-related toxicities.

\section{Discussion}

Primary intestinal amyloidosis is a very rare cause of acute small intestinal pseudo-obstruction, which physicians may not think of when the patient has no predisposing factors. Our patient presented with an uncommon etiology of acute small intestinal obstruction as the first clinical presentation of intestinal amyloidosis.

Amyloid deposition in the GI tract is the greatest in the small intestine, with $31 \%$ of patients with amyloidosis discovered at autopsy [2]. The disease may be diffuse or rarely localized. Clinical manifestations of intestinal amyloidosis may vary from asymptomatic to serious forms. The reported luminal GI presentations included abdominal pain (50\%), change of bowel habits (47\%), altered gut motility (39\%), spontaneous bowel perforation (16\%), and GI bleeding (39\%) [3]. The most common systemic symptoms are weakness, fatigue, purpura, autonomic dysfunction, and marked unexplained weight loss. Cardiac, neuromuscular, renal, dermatologic, and GI tract involvement are common in AL amyloidosis [2]. Amyloidosis may affect the GI system in isolation or present with multisystem involvement. However, the diagnosis is often delayed due to the vague clinical presentation.

Pseudo-obstruction is not usually reported in extreme cases of AL amyloidosis in which the pathomechanism is intestinal dysmotility. Amyloid infiltration between the muscle fibers of the intestinal smooth muscle causes pressure atrophy of the adjacent fibers. The involvement of the myenteric plexus by amyloid deposition and vascular insufficiency results in hypomotility of the affected intestine [4]. These patients usually have chronic obstructive symptoms and acute phases of pseudo-obstruction as illustrated in our case. The vasculature of the small intestine is usually involved, with a high risk of ischemia and infarction in case of narrowing and occlusion of the vessels.

Radiological findings typically present as the clinical features of mechanical obstruction with plain films showing a paralytic ileus. These patients usually have chronic obstructive symptoms and acute phases of pseudo-obstruction. Computed tomography scans may show small bowel thickening and dilatation with a "double halo" appearance [5].

Endoscopic appearance of AL amyloidosis typically forms polypoid lesions, mucosal protrusions, and thickening of the valvulae conniventes, while AA amyloidosis is associated more with diffuse deposition causing mucosal friability and ulcerations [4]. As a result, AL amyloidosis usually presents with constipation, mechanical obstruction, or chronic intestinal pseudoobstruction, while AA amyloidosis presents with diarrhea and malabsorption [6].

Biopsy-proven primary light-chain amyloidosis involving the GI tract is rare. It has been reported that only 3.2\% had biopsy-proven amyloid involvement of the GI tract [7]. The organ to be biopsied in order to diagnose amyloidosis has classically been the rectum or subcutaneous fat. Submucosal vessels should be sampled in rectal biopsies in order to get the reported yield of 75-94\%. Biopsies of the kidney, subcutaneous fat, small intestine, and liver result in over $80 \%$ yield [2]. AL amyloidosis is a diagnosis of exclusion and the demonstration of 
monoclonal gammopathy supports the diagnosis. A radiographic skeletal survey and bone marrow biopsy should be obtained to search for multiple myeloma.

Histologically, amyloid appears homogeneous and amorphous under light microscopy. It stains pink with hematoxylin and eosin and displays metachromasia with methyl violet. Congo red is the most specific stain which produces the characteristic red appearance in normal light and apple-green birefringence in polarized light.

The goal of treating $\mathrm{AL}$ amyloidosis is to suppress the abundance of the amyloidogenic precursor protein which leads to the improvement of amyloidotic organ dysfunction. The first-line recommended treatment is combination chemotherapy regimens that include highdose chemotherapy with melphalan and dexamethasone and hematopoietic stem cell transplantation. Proteasome inhibitor-based regimens (bortezomib-alkylator steroid combination) are the preferred choice due to better response rates and outcomes [8, 9]. Prokinetic agents may benefit dysmotility-related symptoms. Supportive measures such as total parenteral nutrition and antidiarrheal agents can be beneficial.

Pseudo-obstruction due to amyloidosis carries a particularly grave prognosis. The prognosis for patients with focal intestinal amyloidosis is generally good. However, the prognosis in patients with a systemic disease is usually poor. All patients died in a case series of systemic AL amyloidosis presenting with chronic intermittent obstructive symptoms with amyloid infiltrating smooth muscle [4]. If AL amyloidosis is treated with melphalan and prednisone, the prognosis is dismal with a median survival of less than 2 years. In patients who underwent hematopoietic stem cell transplantation, the 5-year survival rate was about $60 \%$ [10].

In conclusion, the clinical manifestations and radiological findings as illustrated in our case can be very suggestive of acute mechanical obstruction that mimics the indication for surgical intervention but would be of no benefit. Recognition of primary intestinal AL amyloidosis in patients with unexplained etiologies of luminal obstruction is important in order to avoid laparotomy. Once amyloidosis is considered, the diagnosis can be obtained by biopsy of the rectal or small bowel mucosa or even by biopsy of subcutaneous adipose tissue, which might be positive in many patients with systemic amyloidosis.

\section{Acknowledgements}

The authors acknowledge Prof. Winyou Mitranun and Dr. Poowadon Wetwittayakhlung for providing histological pictures for this case.

\section{Statement of Ethics}

Informed consent was obtained for this case report. The name of the subject in this case is not mentioned in the case report as well as other protected health information (PHI) and no identifiable image has been included with this case.

\section{Disclosure Statement}

The authors have no conflicts of interest to declare.

\section{Funding Sources}

There are no funding sources to report. 
Wetwittayakhlang et al.: Primary Gastrointestinal Amyloidosis: An Unusual Cause of Acute Intestinal Pseudo-Obstruction

\section{Author Contributions}

P. Wetwittayakhlang performed data acquisition and drafted the manuscript, P. Sripongpun and S. Jandee supervised and provided critical revision of the manuscript. All authors read and approved the final manuscript.

\section{References}

1 Sattianayagam PT, Hawkins PN, Gillmore JD. Systemic amyloidosis and the gastrointestinal tract. Nat Rev Gastroenterol Hepatol. 2009 Oct;6(10):608-17.

2 Ebert EC, Nagar M. Gastrointestinal manifestations of amyloidosis. Am J Gastroenterol. 2008 Mar;103(3):776-87.

3 James DG, Zuckerman GR, Sayuk GS, Wang HL, Prakash C. Clinical recognition of Al type amyloidosis of the luminal gastrointestinal tract. Clin Gastroenterol Hepatol. 2007 May;5(5):582-8.

4 Tada S, Iida M, Yao T, Kitamoto T, Yao T, Fujishima M. Intestinal pseudo-obstruction in patients with amyloidosis: clinicopathologic differences between chemical types of amyloid protein. Gut. 1993 Oct;34(10):1412-7.

5 Georgiades CS, Neyman EG, Barish MA, Fishman EK. Amyloidosis: review and CT manifestations. Radiographics. 2004 Mar-Apr;24(2):405-16.

6 Hokama A, Kishimoto K, Nakamoto M, Kobashigawa C, Hirata T, Kinjo N, et al. Endoscopic and histopathological features of gastrointestinal amyloidosis. World J Gastrointest Endosc. 2011 Aug;3(8):15761.

7 Cowan AJ, Skinner M, Seldin DC, Berk JL, Lichtenstein DR, O'Hara CJ, et al. Amyloidosis of the gastrointestinal tract: a 13-year, single-center, referral experience. Haematologica. 2013 Jan;98(1):141-6.

8 Wechalekar AD, Gillmore JD, Bird J, Cavenagh J, Hawkins S, Kazmi M, et al.; BCSH Committee. Guidelines on the management of AL amyloidosis. Br J Haematol. 2015 Jan;168(2):186-206.

9 Palladini G, Merlini G. What is new in diagnosis and management of light chain amyloidosis? Blood. 2016 Jul;128(2):159-68.

10 Rajkumar SV, Gertz MA. Advances in the treatment of amyloidosis. N Engl J Med. 2007 Jun;356(23):2413-5.
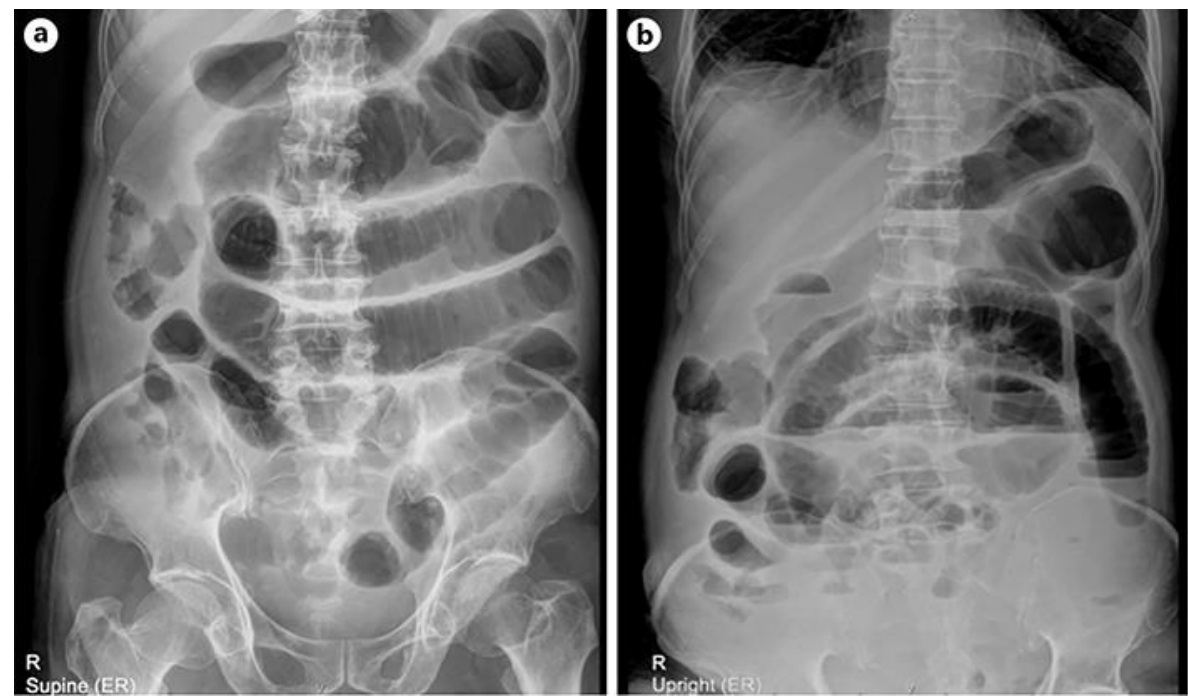

Fig. 1. Plain radiographs of the abdomen: supine position (a) and upright position (b) show dilated bowel loops and multiple air-fluid levels in the small intestine. 


\section{Case Reports in Gastroenterology}

\begin{tabular}{l|l}
\hline Case Rep Gastroenterol 2019;13:462-467 \\
\hline DOI: 10.1159/000503897 & $\begin{array}{l}\text { c 2019 The Author(s). Published by S. Karger AG, Basel } \\
\text { www.karger.com/crg }\end{array}$ \\
\hline
\end{tabular}

Wetwittayakhlang et al: Primary Gastrointestinal Amyloidosis: An Unusual Cause of Acute Intestinal Pseudo-Obstruction
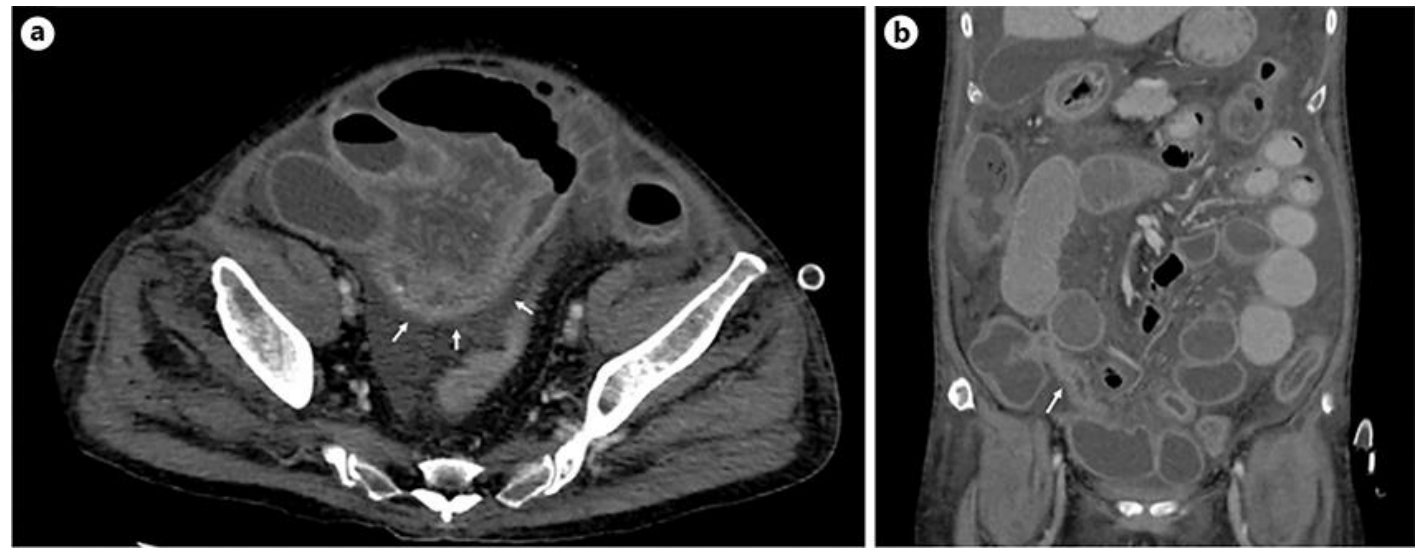

Fig. 2. Computed tomography: axial image (a) and coronal image (b) show evidence of distal small bowel obstruction, which revealed no cause of obstruction, but long segment narrowing of the terminal ileum was seen (arrows).
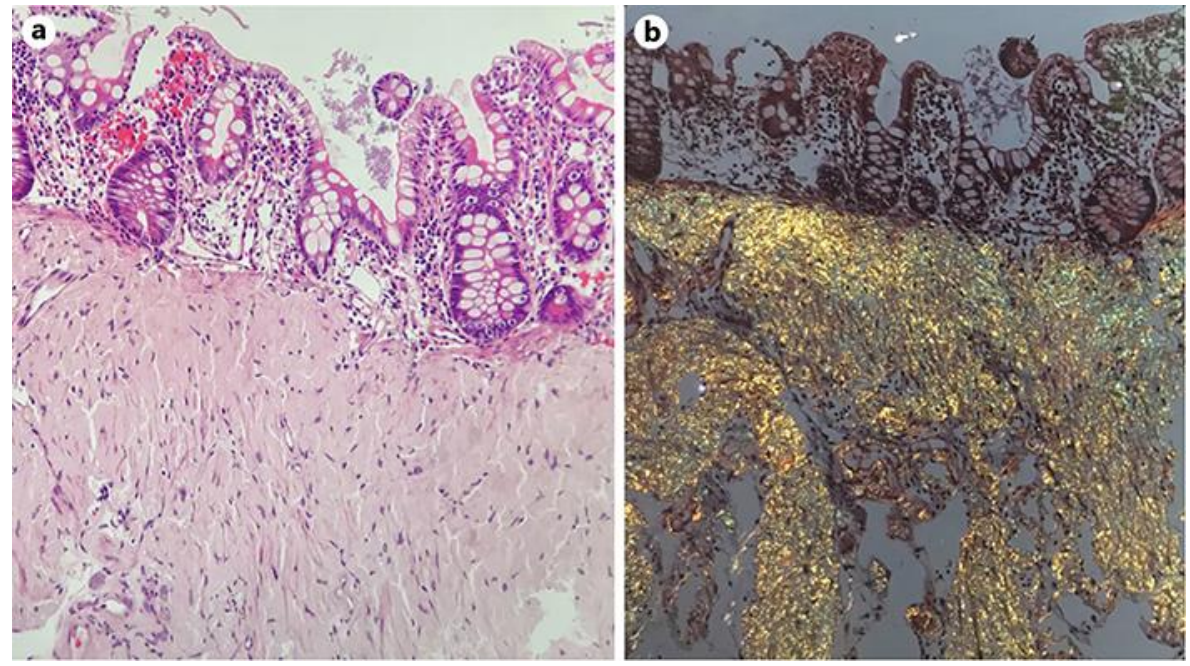

Fig. 3. Pathologic findings of AL type amyloidosis of the luminal gastrointestinal tract. a Histologic examination of a terminal ileum biopsy revealed pink amorphous material diffusely replacing the entire submucosa and involving the blood vessels. Only residual surface lining epithelial cells remained (hematoxylineosin stain; original magnification, 100×). b The same biopsy examined under polarized light with Congo red stain revealed apple-green birefringence, typical of amyloid (original magnification, 100×). 